\title{
Ruxandra Cesereanu \\ La biographie de la censure \\ en Roumanie à travers le regard \\ de trois chercheurs: Adrion Marino, \\ Liviu Malița, Liliana Corobca
}

\section{A BIOGRAPHY OF CENSORSHIP IN ROMANIA:}

AdRIAN MARINO, LIVIU MALIȚA, LILIANA COROBCA

Abstract: This study focuses on three authors

whose research concerns have centered almost

ritualistically on censorship in Romania: Adrian

Marino, Liviu Malița and Liliana Corobca. While

other works and researchers in the field of

censorship studies are also mentioned in this

essay, these three scholars are remarkable in terms of their consistent, ample research on this topic. Each of them has engaged, in their own particular ways, in outlining a history and biography of censorship in Romania. My reasons for selecting these three authors are of a multigenerational nature. Marino, Malița and Corobca belong to very different generations, separated by many years, yet they encapsulate the defining features of their own generation. Keywords: Censorship; History; Romania; Adrian Marino; Liviu Malița; Liliana Corobca.

\section{RUXANDRA CESEREANU}

Université Babes-Bolyai, Cluj-Napoca, Roumanie RuxCes@yahoo.com
T a présente étude vise à prendre en Lcharge trois auteurs qui sont concernés de façon rituelle par la recherche sur la censure en Roumanie : Adrian Marino, Liviu Malița, Liliana Corobca. Sans négliger d'autres livres et d'autres chercheurs dans le domaine, les trois auteurs mentionnés précédemment ont eu des préoccupations constantes sur le sujet de la censure, dans des cadres plus larges et, grâce à leur ambition ils ont abouti (chacun à sa manière) à réaliser une sorte d'histoire et de biographie de la censure en Roumanie. Derrière notre choix il $\mathrm{y}$ a aussi une raison multi-générationnelle car Marino, Malița et Corobca sont les représentants de trois générations très différentes, non seulement à cause des nombreuses années qui les séparent, mais aussi à cause des caractéristiques de la génération que chacun d'entre eux représente. En les rassemblant ici, dans cet album d'analyse critique de la censure, de manière biographique, mon intention a été également de présenter un tableau clinique de la censure à travers trois styles différents d'écriture. 
1.

L e critique littéraire, l'herméneute, l'historien des idées Adrian Marino (19212005) a préféré dans sa dernière période de vie et d'étude être considéré comme un critique des idées du type libéral des Lumières - c'est ainsi qu'il préférait se surnommer. Ses livres publiés après la chute du communisme : Pentru Europa (Pour l'Europe), Polirom, 1995 ; Politică și cultură (Politique et culture), Polirom, 1996 ; Revenirea în Europa (Retour à l'Europe), Aius, 1996, mais surtout ceux qui portent sur la liberté et la censure : Cenzura în România. Schiță istorică introductivă (La censure en Roumanie. Esquisse historique introductive), Aius, 2000 ; Libertate și cenzură în România (Liberté et censure en Roumanie), Polirom, 2005, défendent cette position.

Cenzura în România. Schiță istorică introductivă est la version roumaine d'un texte proposé par l'auteur dans une encyclopédie britannique sur la censure ; par conséquent, le texte a le mérite d'être une synthèse condensée, introductive et informative. Le mérite du livre d'Adrian Marino est de faire l'histoire de la censure sur les territoires roumains, avec un regard sobre et précis (toutefois, non sans des observations croustillantes par rapport à la phobie de la censure) et d'examiner, sur différentes ramifications avérées, la typologie de la censure religieuse et politique pendant trois siècles.

Ainsi le XVII ${ }^{\text {ème }}$ siècle est dominé par la censure religieuse d'un point de vue catholique et orthodoxe. En Transylvanie la censure a une forme centralisée, laïcisée, bureaucratisée et quatre types d'écriture sont interdits (l'écriture pornographique, celle magique/païenne c'est-à-dire antichrétienne, et celle politiquement dangereuse). La Valachie et la Moldavie sont plus libérales pendant cette période, même si la censure religieuse orthodoxe fonctionne vigoureusement contre les écritures hérétiques, athées ou rivales (catholiques).

Au cours du XIX ${ }^{\text {ème }}$ siècle, la censure en Transylvanie était brutale, au niveau tant ecclésiastique que politique. Les cas spectaculaires de certains auteurs censurés, qui sont ensuite devenus eux-mêmes des censeurs, retiennent l'attention, car ce sont des noms célèbres tels que Samuel Micu Klein ${ }^{1}$ ou Petru Maior ${ }^{2}$. Dans les autres territoires roumains, la censure connaît une période d'apogée, puis le déclin, la censure étant supprimée en 1866 par la Constitution. A son apogée, la censure connaît néanmoins des formes extrêmement dures à cause du régime de domination russe, qui consacre une période sombre. Le XIX ${ }^{\text {ème }}$ siècle est caractérisé par une collaboration entre le politique et la religion telle que l'auteur l'établit. La révolution de 1848 et les manifestations implicites ont catalysé la première abolition officielle de la censure, mais après la suppression du boom démocratique jusqu'à l'Union de 1859 , la censure a connu un renouvellement et a reçu une coloration presque totalitaire. Il faut mentionner, par exemple, les procédures de presse contre certaines personnalités, telles que Alexandru Macedonski ${ }^{3}$ (pour le pamphlet) et BP Hasdeu ${ }^{4}$ (pour la prose licencieuse). En Transylvanie, George $\mathrm{Barit}^{5}$ est celui qui dénonce furieusement le côté répressif de la censure, et Ioan Slavici ${ }^{6}$ est l'écrivain qui subit le plus d'agressions (y compris la détention) pour ce que l'on appelle le crime de presse.

Le $X^{\text {ème }}$ siècle se caractérise à nouveau par la répression, la libéralisation et 
une fois de plus la répression. En Valachie, les processus des germanophiles Ioan Slavici et Tudor Arghezi ${ }^{7}$, accusés d'avoir collaboré avec l'occupant allemand, résonnent. Le texte de Geo Bogza ${ }^{8}$, Poemul invectivă (Le poème invective), est interdit pour outrage contre les bonnes mœurs, et le roman Domnișoara Cristina (Mademoiselle Christina) de Mircea Eliade ${ }^{9}$ est accusé de pornographie!

$\mathrm{La}$ censure totalitaire au $\mathrm{XX}^{\mathrm{e}}$ siècle comporte trois étapes : royaliste (sous Charles $\mathrm{II}^{10}$ ), antonescienne (sous Ion Antonescu ${ }^{11}$ ) et communiste, cette dernière étape étant la plus dure. Lors de l'invasion russe du 30 août 1944 (l'occupation a duré quatorze ans, jusqu'en 1958 quand les troupes soviétiques se sont retirées de Roumanie), Adrian Marino a perçu une forte résurrection de la censure russe de 1831. La censure communiste s'est révélée bureaucratisée et extrêmement répressive, étant une composante essentielle du système totalitaire. Les épurations des journalistes, la création du fonds secret des bibliothèques publiques, l'interdiction des publications théoriques, tout cela matérialise une censure méticuleusement organisée. Le texte de synthèse d'Adrian Marino fait preuve d'une documentation appliquée, traitée de manière systématique et rigoureuse. Le style est clair, sans des détails inutiles, l'auteur voulant réaliser une biographie de l'idée de censure en Roumanie.

Les deux concepts du deuxième ouvrage livre consacré à la censure, Libertate și cenzură în România, ont fasciné Adrian Marino pendant sa dernière période, l'auteur en regroupant ce couple antithétique, précisément pour mettre en évidence les avantages et les inconvénients de la société et de la culture roumaine, et les a analysés dans un résumé précis, destiné à des applications aux XVIII ${ }^{\text {ème }}$ et XIX $^{\text {ème }}$ siècles.

Adrian Marino dissèque élégamment la pensée politique et la culture roumaine pré-moderne, étant fasciné par l'évolution d'un esprit critique. Obsédé par le lent processus d'européanisation de la culture roumaine, Adrian Marino se concentre surtout sur le concept de modernité appliqué dans le domaine de la pensée politique. En ce qui concerne la censure, il préfère, grâce à la relation synonymique, le terme coercition, parfois jugé plus adéquat vu la nocivité évidente de son sens. Malheureusement, sa disparition physique en 2005 a empêché Adrian Marino d'achever le dernier chapitre de son œuvre, à savoir « La confrontation entre libéralisme et les totalitarismes de droite et de gauche ».

En ce qui concerne le titre de cet ouvrage, il convient de noter que l'intérêt et la fascination de l'auteur sont surtout liés à l'idée de liberté et non à la censure. Il n'y avait aucune défense idéologique pro domo pour la liberté jusqu'à ce que la contrainte apparaisse. La Transylvanie a été le premier territoire roumain où l'idée de liberté a commencé à être débattue de manière professionnelle en ayant un cadre idéologique. La discussion est restée quand même dans le milieu élitiste et n'est pas parvenue à intégrer celui de la Roumanie profonde, des masses. Liidée de liberté comporte des nuances différentes en Transylvanie et dans les autres pays roumains, mais ce point est prévisible et entièrement justifiable. La principale observation d'Adrian Marino est néanmoins autre : que l'idée de liberté, telle qu'elle apparaît en Transylvanie, est entièrement contemporaine des mouvements des idées d'Europe, au moins ce 
point prouvant qu'au niveau du XVIII ${ }^{\text {ème }}$ siècle, nous étions parfaitement synchronisés avec l'Europe et intégrés à celle-ci. Même si l'Europe ne nous connaissait pas!

Une chronologie de l'idée de liberté est pourtant nécessaire ; on apprend ainsi que Paul Iorgovici ${ }^{12}$, en 1799 , a été le premier à exprimer clairement l'idée en question, suivi de près par Samuil Micu et les autres voix de l'École de Transylvanie. L'analyse d'Adrian Marino est faite petit à petit, en détail, pour illustrer un panorama complet du Zeitgeist de lépoque. La plupart des interventions liées à l'idée de liberté apparaissent dans les débats théologiques ou linguistiques, puis dans ceux historiques et socio-politiques. Lidée de liberté est cependant résolument engagée en Transylvanie par l'aspiration à la liberté de religion, et seulement après par la liberté socio-politique (l'idée nationale) et par la liberté de la presse. Supplex Libellus Valachorum ${ }^{13}$ est une quintessence surtout des deux premiers points. L'idée ou les idées européennes pénètrent la Valachie et la Moldavie à travers la Transylvanie, c'est la démonstration d'Adrian Marino : la Transylvanie est le premier espace roumain qui assimile le modèle européen et découvre l'Europe à travers la circulation des idées.

Des études de cas pareils sont consacrées à la Moldavie et à la Valachie, mais la Transylvanie reste, au moins pour le $\mathrm{XVIII}^{\text {ème }}$ et le début du XIX ${ }^{\text {ème }}$ siècle, la championne de l'européanisation de l'espace roumain et de la familiarisation avec les idées qui circulent en Europe. Les deux autres pays roumains sont considérés comme moins impétueux dans la promotion de l'idée de liberté, au moins pendant la période exacte sur laquelle l'auteur mène son enquête. L'essor en Moldavie et en Valachie est individuel et non collectif (comme en Transylvanie), et les influences ont leur origine dans les filières phanariotes et russes, et non dans celles occidentales. Cependant, le développement de l'esprit critique et le processus de modernisation se poursuivent progressivement et de manière bénéfique dans le cas de la Moldavie et de la Valachie, deux régions plus traditionnellement patriarcales que la Transylvanie. Les curiosités ne manquent pas : en Moldavie, la franc-maçonnerie inculque un courant d'idées libérales-occidentales ; il est important donc de souligner le manque de censure et l'importation massive de livres étrangers, approuvés par les dirigeants cosmopolites étrangers ; enfin et surtout, l'impact et les échos de la révolution française sont essentiels. Le cas de la Valachie est presque identique à celui de la Moldavie ; l'auteur observe quand même un processus d'européanisation plus intense qu'en Moldavie même si le risque était de déclencher des «formes sans fond ». Un autre bloc impressionnant est dédié au début du XIX ${ }^{\text {ème }}$ siècle en Transylvanie, une véritable obsession de l'auteur, je dirais, de fixer les origines du processus d'européanisation de la culture roumaine dans l'espace de la Transylvanie, et non dans celui du sud ou de la Moldavie.

\section{2.}

T iviu Malița (né en 1959) a commencé Lses recherches sur la censure en 2006, avec deux volumes collectifs qu'il a coordonnés dans le domaine théâtral : Viața teatrală in și după comunism (La vie théâtrale pendant et après le communisme), et Cenzura in teatru. Documente (La censure 
dans le théâtre. Documents), 1948-1989, tous les deux parus aux éditions EFES. Le premier volume comprend divers dossiers de spectacle et quatre études sur la transition du théâtre roumain du communisme au postcommunisme ; le deuxième volume est une collection de documents provenant de diverses archives, organisés par thèmes et $\mathrm{du}$ point de vue chronologique et investigués à travers les méthodes de l'histoire orale. Dix ans plus tard, en 2016, Liviu Malița a publié deux volumes individuels sur la censure : Cenzura pe înțelesul cenzuraților (La censure racontée aux censurés), paru chez Tracus Arte et Literatura eretică. Texte cenzurate intre 1949-1977 (La littérature hérétique. Textes censurés entre 1949-1977), paru aux éditions Cartea Românească. Il s'agit de deux amples volumes d'analyse des mentalités ; liés au phénomène de la censure, ce sont des monographies de la répression artistique, montrant une recherche approfondie de la manipulation par la propagande sous le régime communiste en Roumanie. Les deux livres sont orwelliens, chacun à sa manière, en ce qui concerne la mentalité : les analyses et simultanément les synthèses faites par Liviu Malița reflètent la manière dont, pendant la période communiste, la censure n'était pas seulement le superviseur drastique des cerveaux artistiques, mais elle était même devenue l'environnement de production de la culture. La censure est devenue précisément la matrice qui forgeait la culture, et surtout la littérature, d'une manière procustienne. La recherche de Liviu Malița présente une progression nuancée de la censure de la période classique jusqu'à la néo-censure (après 1977) quand la Direction de la Presse et des Imprimés a été dissolue, mais la censure a continué de fonctionner de manière punitive en catalysant même l'autocensure des auteurs impatients de publier, mais prudents quant à leur propre littérature au risque d'une possible subversion. Les livres de Liviu Malița provoquent une lecture à deux niveaux : au niveau normal (en haut de page), il y a les principales analyses, mais tout aussi captivantes sont les notes de bas de page qui représentent un autre niveau de lecture, plus aigu, nuancé et parfois même interprétatif.

Les origines de la censure dans le communisme sont dérivées de quelques modèles reconnus : la censure soviétique bolchevique, mais aussi la censure royale carliste. Le premier livre (Cenzura pe întelesul cenzuraților) contient également le dossier d'un manuel commenté sur la censure, puisque le manuel présente clairement les règlements, les normes et la méthodologie, les principales actions de la censure (interdiction, mutilation, persuasion), des portraits typologiques de censeurs et de ceux censurés (de diverses nuances intermédiaires - censures complices, par exemple, ou écrivains ayant une position institutionnalisée). Liviu Malița analyse tous ces éléments en tant que législateur, avec une prégnance et une flexibilité juridique remarquables. La mise en scène du fonctionnement de la censure est emblématique pour le fonctionnement de ce commando institutionnel : 1. 1949-1954, période de guerre, de gardien brutal, de censure ; 2 . 1954-1969, période de censure pédagogique et « didactique »; 3. 1971-1977, la période de restalinisation de la censure, de la mise en œuvre de l'idée de purification idéologique et de contrôle extrême. L'ensemble du système de censure ramifié est radiographié au niveau décisionnel. 
Une section spéciale est occupée par les portraits des censeurs et des censurés. Typologiquement parlant, les censeurs sont des agents de l'interdiction et des nettoyeurs à tendance (feux rouges des déraillements, des insuffisances, des hérésies) selon une hiérarchie bien établie. Les censeurs sont, dans le sens psycho-historique, des soldats linguistiques ou des tortionnaires des mots, des gardes du corps du parti. Et les censurés sont de « potentiels criminels " qui doivent être étroitement surveillés et ensuite guidés afin de ne pas commettre des erreurs idéologiques. La recherche de Liviu Malița présente une série d'études de cas sur les politiques éditoriales et journalistiques et sur la pratique de la censure dans la Roumanie communiste, en coagulant un manuel de procédure sur l'interventionnisme abusif de la censure. La manifestation indique clairement que la censure était une méthode de punition et de contrôle de la police politique (Securitatea).

Cenzura pe ințelesul cenzuratilor comprend également des scènes d'un humour absurde, des séquences sur les ragots officiels entre les censeurs et sur les traîtres parmi eux, car ces fonctionnaires nont jamais été rigides, mais vicieux, blâmables, voire névrotiques, blasés, et certains (très peu) y compris relativement rebelles quand ils n'étaient pas carriéristes et robotisés (dans la plupart des cas). Les déraillements de la typologie robotique du censeur indiquent le fait que le système ne fonctionnait pas parfaitement, bien que son pourcentage de monstruosité fût énorme et l'ivresse du pouvoir, extrême.

Ce n'était pas forcément l'acte qui devait être puni, mais le cerveau " mal pensant » de certains écrivains et artistes perçus comme rebelles, d'où la typologie des censurés : écrivains officiels, écrivains tolérés, écrivains interdits. La censure devient pendant le communisme une discipline répressive visant à découvrir et à éliminer dans les œuvres littéraires, non seulement des faits et des scénarios idéologiques indésirables par le système, mais aussi des intentions malveillantes qui devaient être sanctionnées avant de se répercuter collectivement.

Literatura eretică. Texte cenzurate intre 1949-1977, le livre qui continue la recherche de La censure racontée aux censurés défend l'idée comme quoi pour les censeurs, l'hérétique était n'importe qui pourrait être blâmé officiellement, d'où le caractère castrateur, étouffant, défigurant et vigilent-stérilisant de l'institution. Parfois, la censure était déroutante, voire hallucinante, avec des explosions paradoxales de tolérance et de permissivité (pendant les périodes de détente idéologique). Mais généralement, elle a agi pareil à un bolide brutal et destructeur à travers la malformation.

Quels étaient les textes censurables et dangereux ? Tous - id est les textes religieux (seulement les textes qui caricaturaient et ironisaient la religion étaient acceptés), les textes philosophiques, ceux historiques, la littérature des angoisses, les textes évasionnistes, ceux érotiques. C'est la raison pour laquelle le deuxième livre de Liviu Malița, avec ses études de cas, est un manuel-raisonneur sur la chasse et le harcèlement, car tout était dangereux du point de vue de la censure. Les mots, les thèmes, les sujets, les religions, les idéologies, les histoires, etc. ont été chassés. La poésie a été, par exemple, censurée parce qu'elle était métaphysique, asociale et confessionnelle. 
La prose a été censurée parce qu'elle incarnait un pluri-perspectivisme de voix narratives, impossible à contrôler. Le théâtre a été censuré principalement parce qu'il était publiquement représentatif. La diffusion d'un livre était également censurable, selon l'auteur et le thème. Il y avait un certain nombre d'ismes répudiés et taxés par la censure : apolitisme, esthétisme, formalisme, naturalisme, avant-gardisme, onirisme, évasionnisme, hermétisme, expérimentalisme. La censure manifestait une répulsion particulière envers le fantastique et l'absurde qu'il lui était impossible de discipliner, en raison de leur caractère codé et souvent dystopique.

L'histoire de la Roumanie a été la plus surveillée par l'œil de cyclope de la censure. Il était interdit de critiquer le Parti communiste, le communisme, les dirigeants du $\mathrm{PCR}$, mais aussi les légionnaires ${ }^{14}$, la figure d'Ion Antonescu, des territoires comme la Bucovine, la Bessarabie, l'emprisonnement des Roumains en URSS, la démystification des personnages historiques comme Avram Iancu ${ }^{15}$ (déprimé après la défaite de la révolution), les indésirables comme les membres de la famille Brătianu ${ }^{16}$, Iuliu Maniu $^{17}$, les évêques martyrs de l'Église grecque-catholique $^{18}$, le jour du 23 août $1944^{19}$, etc. La religion était complétement interdite. L'actualité était censurée à un niveau descriptif : la banalité quotidienne, le gris quotidien, la pénurie morbide des temps étaient rejetés.

Le cas de Paul Goma ${ }^{20}$ et de son roman, Ostinato, est fascinant et Liviu Malița le présente en détail. Censuré au niveau du manuscrit, Goma accepte initialement diverses interférences et amputations signalées par la censure. Plus tard, après que son cas rebelle est devenu international, Paul Goma a développé précisément les thèmes et les questions interdits par la censure, en les amplifiant. Sa chance a été la publication du roman en Occident et la popularité dont Alexandr Soljenitsyne y jouissait et avec lequel Goma a été comparé positivement.

Liviu Malița analyse également du point de vue psycho-historique la pertinence de la culture de l'ego à laquelle la censure communiste est allergique. La poésie intime et la prose psychologique, en particulier, répugnent aux autorités, car elles ne visent pas la communauté et les masses, mais leur contraire.

Un avant-dernier chapitre du deuxième livre signé par Liviu Malița porte son regard sur la situation de la critique et de l'histoire littéraire pendant le communisme, ces derniers étant considérés comme des outils de purification et de contrôle idéologique à travers le regard des censeurs. L'institution du contrôle visait à transformer les critiques littéraires et les historiens dans une sorte de "procureurs" pendant le premier stade du communisme. Mais, progressivement, la critique littéraire s'est émancipée, a gagné de l'autonomie et est devenue récalcitrante par rapport à la censure, et, par conséquent, les livres de critique littéraire et d'histoire ont commencé, à leur tour, à devenir des matières censurables et des corps du délit.

Le dernier chapitre problématise et met l'emphase sur le statut de la littérature roumaine pendant la période communiste, avec quelques conclusions : 1 . il y a eu une bataille souterraine de tranchées entre censeurs et censurés ; 2 . le samizdat était inexistant dans la Roumanie communiste et la littérature subversive était peu développée ; 3. afin de se défendre, la littérature 
roumaine a montré une prédilection pour les codes pendant le communisme; 4 . le compromis entre l'institution de la censure et les auteurs a été plutôt pragmatique et dans certains cas on pouvait observer différentes formes d'autocensure.

Liviu Malița n'est pas seulement un historien littéraire qualifié comme il le montre dans ses deux livres sur la censure, mais aussi un remarquable analyste des mentalités. Il a la précision d'un justicier lucide, objectif, impétueux, dans un style élevé et souple. Son œil est à la fois analyste et perspectiviste, ayant les caractéristiques d'une loupe minutieuse, mais aussi la capacité de synthèse.

\section{3.}

$U_{\mathrm{d}}^{\mathrm{n}}$

ne troisième chercheuse qui traite de façon systématique le thème de la censure est Liliana Corobca (née en 1975). Liliana Corobca considère que l'institution de la censure était, dans la Roumanie communiste, la troisième fonction essentielle à la matérialisation de la répression, à côté du Parti communiste et de la Securitate. La chercheuse a d'abord publié deux volumes (dans Institutia cenzurii comuniste în România. 1949-1977 [L'institution de la censure communiste en Roumanie. 1949-1977], éditions Ratio et Revelatio Publishing House, 2014) qui contiennent des documents sur la structure, les attributions et le fonctionnement de l'institution de censure, ainsi que des documents sur les relations avec les autres institutions. Dans l'introduction des deux volumes, Liliana Corobca a également résumé le fonctionnement de la censure principalement en URSS, mais aussi dans d'autres États communistes (la Pologne, la Tchécoslovaquie, la Bulgarie, la Hongrie,
l'Allemagne de l'Est, la Yougoslavie). Toujours en 2014 la chercheuse a publié le volume Controlul cărții. Cenzura literaturii in regimul comunist din România (Le contrôle du livre. La censure de la littérature pendant le régime communiste en Roumanie), paru aux éditions Cartea Românească, un livre utile vu qu' il facilite une comparaison et un parallèle entre la censure en URSS (au niveau des critères de purification et non seulement) et celle dans la Roumanie communiste, indiquant la manière dont les autorités roumaines ont imité et assumé le "modèle » soviétique de la censure, de la purification et de la création du fonds (secret) spécial de livre. Pareil à Liviu Malița, Liliana Corobca déconstruit le mécanisme de censure comme un équipement complexe et sophistiqué. La déconstruction est très détaillée, le matériel documentaire est primordial, les citations sont nombreuses. La chercheuse préfère composer et proposer une sorte de fresque épique de la censure, d'où les exemples abondants et les citations tout aussi généreuses des documents lus, qui dominent souvent le texte et le commentaire critique. Le livre se présente non seulement comme une étude idéationnelle de la censure mais surtout comme une étude essentiellement exemplaire, Liliana Corobca s'intéressant à une multitude d'études de cas qu'elle énumère et rassemble pour faciliter, à travers l'exemple, la compréhension du phénomène de la censure.

Il faut mentionner que les approches d'autres chercheurs roumains tels que Ionut Costea, Istvan Kiraly, Doru Radosav (les auteurs du volume Fond Secret. Fond "S" special. Contributii la istoria fondurilor secrete de bibliotecă din România, Studiu de caz. Biblioteca Central Universitară "Lucian Blaga” (Fonds secret. Fonds «S» spécial. Contributions 
à l'bistoire des fonds secrets des bibliothèques en Roumanie. Étude de cas. Bibliothèque centrale de l'Université "Lucian Blaga», 1995), Lidia Vianu (Censorship in Romania [La censure en Roumanie], 1998), Marian Petcu, l'auteur du volume Puterea și cultura. O istorie a cenzurii (Le pouvoir et la culture. Une histoire de la censure), 1999 et le coordinateur du volume collectif Cenzura în spațiul cultural românesc (La censure dans l'espace culturel roumain), 2007 ; Bogdan Ficeac, Cenzura comunistă si formarea "omului nou" (La censure communiste et la création de l' " homme nouveau "), 1999 ; Stelian Tănase - Acasă se vorbește in șoaptă. Dosar și Jurnal din anii târzii ai dictaturii (Chez nous, on chuchote. Dossier et journal des dernières années de la dictature), 2002 ; Cristian Vasile - Literatura și artele in România comunistă (La littérature et les arts dans la Roumanie communiste), 2010 et Politicile culturale comuniste in timpul regimului Gheorghiu-Dej (Les politiques culturelles communistes sous le régime de Gheorghiu-Dej), 2011 ; Paul Caravia - Gândirea interzisă. Scrieri cenzurate. România 1945-1989 (La pensée interdite. Écrits censurés. Roumanie 1945-1989), 2000 ; Marin Radu Mocanu, auteur des volumes Cenzura comunistă (La censure communiste), 2001 ; Literatura română și cenzura comunistă. 1960-1971 (La littérature roumaine et la censure communiste. 1960-1971), 2003 ; Cenzura a murit, trăiască cenzorii - documente (La censure est morte, vivent les censeurs - documents), 2008 ; Tiberiu Troncotă - România comunistă. Propagandă și cenzură (La Roumanie communiste. Propagande et censure), 2006 ; Eugen Negrici - Literatura română sub comunism (La littérature roumaine sous le communisme), 2010 ; Ilie Rad - coordinateur du volume collectif Cenzura în România (La censure en Roumanie), 2012 ; Emilia Șercan - Cultul secretului. Mecanismele cenzurii in presa comunistă (Le culte du secret. Les mécanismes de censure dans la presse communiste), 2015 sont très utiles pour le chercheur intéressé par le sujet de la censure. J'ajouterais également deux écrivains bien connus dont les témoignages directs présentent un grand intérêt pour le sujet de la censure : Norman Manea, Despre clovni: dictatorul și artistul (Les clowns. Le dictateur et l'artiste), 1997 et Ana Blandiana, Fals tratat de manipulare (Faux traité de manipulation), 2013. Ma liste n'est pas complète, seulement emblématique et synthétique. Il ne reste que dans l'avenir, un jeune chercheur (ambitieux) rédige avec soin une éventuelle histoire de la recherche sur la censure en Roumanie qui comprendra de manière exhaustive tous les volumes consacrés à ce sujet.

\section{Traduit en français par Mădălina Timu}

\section{Bibliographie}

Liliana Corobca (ed.), Instituţia cenzurii comuniste în România. 1949-1977, volumes I et II, édition, avant-propos et notes par Liliana Corobca, Oradea, Éditions Ratio et Revelatio, 2014.

Liliana Corobca, Controlul cărții. Cenzura literaturii în regimul comunist din România, București, Éditions Cartea Românească, 2014.

Liviu Malița (coordinateur), Viața teatrală în și după comunism, Studiu introductiv de Liviu Malița, Cluj, Éditions EFES, 2006.

Liviu Malița (éditeur), Cenzura în teatru. Documente. 1948-1989, Avant-propos par Liviu Malița, Cluj, Éditions EFES, 2006. 
Liviu Malița, Cenzura pe înțelesul cenzuraților, București, Éditions Tracus Arte, 2016.

Liviu Malița, Literatura eretică. Texte cenzurate intre 1949-1977, București, Éditions Cartea Românească, 2016.

Adrian Marino, Cenzura în România. Schiță istorică introductivă, Craiova, Éditions Aius, 2000.

Adrian Marino, Libertate și cenzură în România, Iași, Polirom, 2005.

\section{Notes}

1. Samuil Micu (1745-1806), théologien grec-catholique, historien, philologue, lexicographe et philosophe roumain des Lumières, représentant de l'École de Transylvanie.

2. Petru Maior (1756-1821), historien, philologue et écrivain roumain, doyen grec-catholique, représentant de l'École de Transylvanie

3. Alexandru Macedonski (1854-1920), poète, prosateur et publiciste.

4. B. P. Hasdeu (1838-1907), écrivain et philologue, pionnier dans diverses branches de la philologie et de l'histoire roumaines ; académicien, encyclopédiste, juriste, linguiste, folkloriste, publiciste, historien et homme politique.

5. George Bariț (1812-1893), historien, homme politique et journaliste de Transylvanie, fondateur de la presse roumaine de Transylvanie.

6. Ioan Slavici (1848-1925), écrivain, journaliste et enseignant.

7. Tudor Arghezi (1880-1967), poète, prosateur, pamphlétaire, l'un des écrivains modernes essentiels de la littérature roumaine.

8. Geo Bogza (1908-1993), journaliste et poète, théoricien de l'avant-garde.

9. Mircea Eliade (1907-1986), historien des religions, écrivain de fiction, philosophe.

10. Charles II (1893-1953), roi de Roumanie entre le 8 juin 1930 et le 6 septembre 1940 ; fils aîné du roi Ferdinand I ${ }^{\text {er }}$ de Roumanie et de son épouse, la reine Maria.

11. Ion Antonescu (1882-1946), militaire et homme d'État, officier de carrière, chef du bureau des opérations au quartier général de l'armée pendant la Première Guerre mondiale, commandant de l'École supérieure de guerre, chef d'état-major et ministre de la Guerre pendant la Seconde Guerre mondiale, et depuis le 4 septembre 1940 jusqu'au 23 août 1944, Premier ministre de la Roumanie avec le titre de « chef d'État "; il a été exécuté par les communistes en 1946.

12. Paul Iorgovici (1764-1808), homme de culture, juriste, il menait des recherches sur l'origine romaine du peuple roumain, parmi les premières personnes du Banat à avoir écrit en roumain en utilisant l'alphabet latin.

13. Supplex Libellus Valachorum est le mémoire de la nation roumaine de Transylvanie de 1791, l'acte politique le plus important des Roumains de Transylvanie au XVIII ${ }^{\text {ime }}$ siècle. Née dans le contexte des Lumières, la loi couronne de longs efforts pour l'émancipation politique et nationale des Roumains ainsi qu'une longue série de mémoires et de pétitions. Supplex Libellus Valachorum formule de façon synthétique les demandes du peuple roumain et est un document essentiel de la lutte pour l'émancipation nationale.

14. La Garde de fer, le mouvement légionnaire ou la Légion de l'Archange Michel - mouvement nationaliste et antisémite dans la Roumanie de l'entre-deux-guerres, qui a recouru à des tactiques terroristes. Elle a été fondée en 1924 par Corneliu Zelea Codreanu.

15. Avram Iancu (1824-1872), révolutionnaire qui a joué un rôle important dans la révolution de 1848 en Transylvanie. Il était le chef de l'armée des Roumains de Transylvanie, en alliance avec l'armée autrichienne, contre les troupes révolutionnaires hongroises sous la direction de Lajos Kossuth.

16. La famille Brătianu, famille de boyards roumains, dont plusieurs personnalités politiques (d'orientation libérale) de l'histoire de la Valachie et de la Roumanie font partie.

17. Iuliu Maniu (1873-1953), homme politique, plusieurs fois Premier ministre de la Roumanie pendant l'entre-deux-guerres, président du Parti national-paysan ; prisonnier politique après 1947, il meurt dans la prison communiste de Sighet. 
18. L'Église grecque-catholique ou l'Église roumaine unie à Rome est l'une des deux églises nationales roumaines. Elle a été dissoute par le régime communiste en 1948 et ses évêques ont été arrêtés, la plupart d'entre eux étant morts dans des prisons ; pour les autres des enquêtes rigoureuses et une résidence obligatoire ont suivi. Il s'agit de Valeriu Traian Frențiu, Vasile Aftenie, Ioan Suciu, Tit Liviu Chinezu, Ioan Bălan, Alexandru Rusu et Iuliu Hossu. L'Église grecque-catholique a été rétablie en 1990 et les sept évêques martyrs ont été béatifiés par le pape François le 2 juin 2019, sur la plaine de la liberté de Blaj. 19. Le 23 août 1944, un coup d'État a eu lieu en Roumanie, à travers lequel le roi Michel I ${ }^{\text {er }}$ a voulu renverser la dictature du maréchal Ion Antonescu et retirer le pays de l'alliance avec l'Allemagne nazie. Cela a raccourci la durée de la Seconde Guerre mondiale et a permis à la Roumanie de s'allier, dans la dernière étape de la guerre, avec les puissances occidentales gagnantes. Moins d'un an après ce coup d'État du roi Michel I ${ }^{\text {er }}$, la dictature d'Antonescu a été remplacée par la dictature communiste. L'historiographie communiste de 1945 à 1989 a nié le rôle essentiel du roi Michel I ${ }^{\text {er }}$, en affirmant à tort que le moment du 23 août 1944 avait été pensé et réalisé exclusivement par les communistes. Après l'effondrement du régime communiste en Roumanie, c'est-à-dire depuis 1990, la vérité historique liée au rôle essentiel du roi Michel I Ir a été restaurée.

20. Paul Goma (1935-2020), écrivain et opposant anticommuniste. Il a été détenu politique sous le régime de Gheorghiu-Dej et pendant le régime de Ceausescu. Il a été expulsé par les autorités communistes de Roumanie en France en 1977, où il a vécu jusqu’à sa mort. 